\title{
A narrative review of lung cancer cytology in the times of coronavirus: what physicians should know
}

\author{
Pasquale Pisapia $^{1}$, Umberto Malapelle ${ }^{1}$, Maria Salatiello ${ }^{1}$, Rafael Rosell ${ }^{2}$, Giancarlo Troncone $^{1}$ \\ ${ }^{1}$ Department of Public Health, University of Naples Federico II, Naples, Italy; ${ }^{2}$ Cancer Biology and Precision Medicine Program Catalan Institute of \\ Oncology; Germans Trias i Pujol Health Sciences Institute and Hospital Badalona, Barcelona, Spain \\ Contributions: (I) Conception and design: All authors; (II) Administrative support: R Rosell, G Troncone; (III) Provision of study materials or patients: \\ All authors; (IV) Collection and assembly of data: All authors; (V) Data analysis and interpretation: All authors; (VI) Manuscript writing: All authors; \\ (VII) Final approval of manuscript: All authors. \\ Correspondence to: Giancarlo Troncone. Department of Public Health, University of Naples Federico II, Via Sergio Pansini 5, 80131 Naples, Italy. \\ Email: giancarlo.troncone@unina.it.
}

\begin{abstract}
In the modern era of personalized and precision medicine, lung cancer management needs to be carried out in a multidisciplinary manner. Among other disciplines, also cytopathology is key in diagnosis and treatment management of these patients. Indeed, cytopathology specimens are often the only source of available tissue material for morphological diagnosis and molecular purposes in order to guarantee an adequate treatment decision making, since surgical resection specimens are not available when lung cancer is diagnosed at advanced disease stages. Today, as an effect of the current severe acute respiratory syndrome Coronavirus 2 (SARS-CoV-2) pandemic, cytopathology is reorganizing and reshaping many of its procedures and workflows, in order to ensure the safety of cytopathologists and laboratory personnel. In particular, careful attention should be paid on biosafety procedures when pulmonary cytological specimens are handled. In addition, also molecular cytopathology, that provides relevant information on the molecular status and on the potential sensitivity to target treatments, is undergoing major changes. In this setting, fully automated technologies, requiring minimal hands-on work, may be a valid option. The aim of this narrative review is to keep updated all the different professional figures involved in lung cancer management and treatment on how SARS-CoV-2 is modifying lung cancer cytopathology.
\end{abstract}

Keywords: Coronavirus disease 19 (COVID-19); severe acute respiratory syndrome Coronavirus 2 (SARS-CoV-2); rapid on site evaluation (ROSE); lung cancer; cytopathology

Submitted Jun 30, 2020. Accepted for publication Aug 12, 2020.

doi: $10.21037 /$ tlcr-20-795

View this article at: http://dx.doi.org/10.21037/tlcr-20-795

\section{Introduction}

The care of any single lung cancer patient requires an integrated approach and a thoroughly collegial discussion taking place at the institutional lung cancer multidisciplinary team (MDT) table to ensure a timely diagnosis, a complete staging, adherence to international guidelines and opportunities for clinical trial recruitment $(1,2)$. In the MDT, together with oncologists, surgeons, pneumologists, radiologists, molecular diagnosticians and clinical bioinformaticians, also cytopathologists play a central role (3). In advanced disease stages, when tumor surgical resection for histological evaluation is not feasible, cytopathology specimens, including both exfoliative and fine needle aspirative biopsy samples, might be the only source of tissue for a complete and fully autonomous diagnosis, including predictive biomarker testing (4-8). Today, in the times of severe acute respiratory syndrome coronavirus 2 (SARS-CoV-2) pandemic, a number of strictly necessary biosafety procedures are changing the way we practice 
cytopathology (9). The aim of this review is to briefly update the different MDT professional figures, on these new rules and in particular on those requirements to process fresh cytopathological samples, to perform rapid on site evaluation (ROSE), to employ liquid based cytology (LBC) methodology and to provide relevant information on the molecular status of lung cancer $(10,11)$. We present the following article, as a result of a deep analysis of the recent international literature published in English on PUBMED in the last six months focused on the impact of SARS-CoV-2 on the management of lung cancer cytological samples, in accordance with the Narrative Review reporting checklist (available at http://dx.doi. org/10.21037/tlcr-20-795).

\section{Cyto-preparation: biosafety procedures}

According to the international consensus, SARS-CoV-2 is classified as a hazard group 3 organism, being able to cause severe human diseases, representing a serious hazard to employees and community and currently lacking effective prophylaxis and treatment (12-15). Processing pulmonary cytological specimens, including sputum, bronchoalveolar lavage (BAL) and washing, fine needle aspiration (FNA) and pleural effusion, requires different levels of laboratory biosafety measures (Figure 1A) $(15,16)$ These should be able to mitigate the not negligible risk of handling fluids and cells potentially carrying viable and thus transmissible SARS-CoV-2 (16). However, cytopathology diagnostic activities are less dangerous than viral culture, isolation or neutralization assays; thus, procedures equivalent to biosafety level (BSL) 2 are generally sufficient $(14,15,17)$. As a matter of the facts, pleural effusions were processed during the healthcare emergency in our laboratory without reduction (18). Nevertheless, the risk assessment depends on whether cytological samples are properly fixed or are unfixed and fresh. Usually cytological fluids, received unfixed at the laboratory, are mixed with $>70 \%$ alcohol solution in a range from 1:1 to $2: 1$; the virus is inactivated and these specimens can be considered as a low-risk samples and processed according to good microbiological practices and procedures (GMPP) (19). These latter include the use of appropriate disinfectants with proven activity [including, hypochlorite (bleach), alcohol, hydrogen peroxide and phenolic compounds] against SARS-CoV-2 in any laboratory area and surface $(16,19)$. However, immediate fixation may not well preserve morphological details; to improve cyto-preparation quality, cytological fluids can be centrifuged fresh; then, the cell pellet is used to prepare smears, cytospins and cell blocks (20). These procedures carried out on fresh and unfixed cells, however, require appropriate personal protective equipment (PPE), to be worn by all laboratory personnel handling these specimens and a number of more stringent BSL-3 measures (21). Centrifuges should have safety buckets or sealed rotors and should be placed in a biosafety cabinet (BSC). This is key to contain aerosols and respiratory droplets (14). Any procedure initial processing (before inactivation) of all specimens, should take place in an appropriately maintained and validated BSC, in particular when there is the potential to generate aerosols such as slides smearing and air-drying, removing tube caps, blending, shaking, mixing, vortexing, pipetting, aliquoting, and diluting $(9,17)$. In particular, class II BSC are preferable since provide not only personnel and environmental protection but also product protection $(9,17)$. As an alternative class I BSC, frequently used in pathology laboratories, with high efficiency particulate air (HEPA) filters are also appropriate for risk mitigation $(9,17)$. Cytological samples should be delivered by hand rather than by pneumatic tube systems; in fact, pneumatic pods propelled by air pressure may generate aerosols in the case of sample leaking and specimens from suspected SARS-CoV-2 positive patients require a triple leak-proof package (22).

Persistence of viable and thus transmissible SARS$\mathrm{CoV}-2$, due to suboptimal fixation of cells obtained from the oropharyngeal and respiratory tract and processed as LBC samples, represents an additional relevant biosafety issue that needs to be mitigated $(23,24)$. Indeed, LBC methodology is widespread in many laboratories and very often used to prepare respiratory cytological specimens. The reasons are several. First, respect to conventional smears, that may display large amounts of obscuring mucus and/or blood, in the LBC preparations, cells are rinsed in fixative solutions rich in mucolytic and haemolytic agents obtaining slides with a clean background, devoid of obscuring blood and exudates elements (25). Secondly, when a pathologist is not available to prepare on site, LBC simplifies the handling of cytological material, avoiding the need of training interventional radiologists and/or bronchoscopists to prepare high-quality smears (26). Thirdly, LBC limits the exposure to potentially infected fresh cells obtained from 


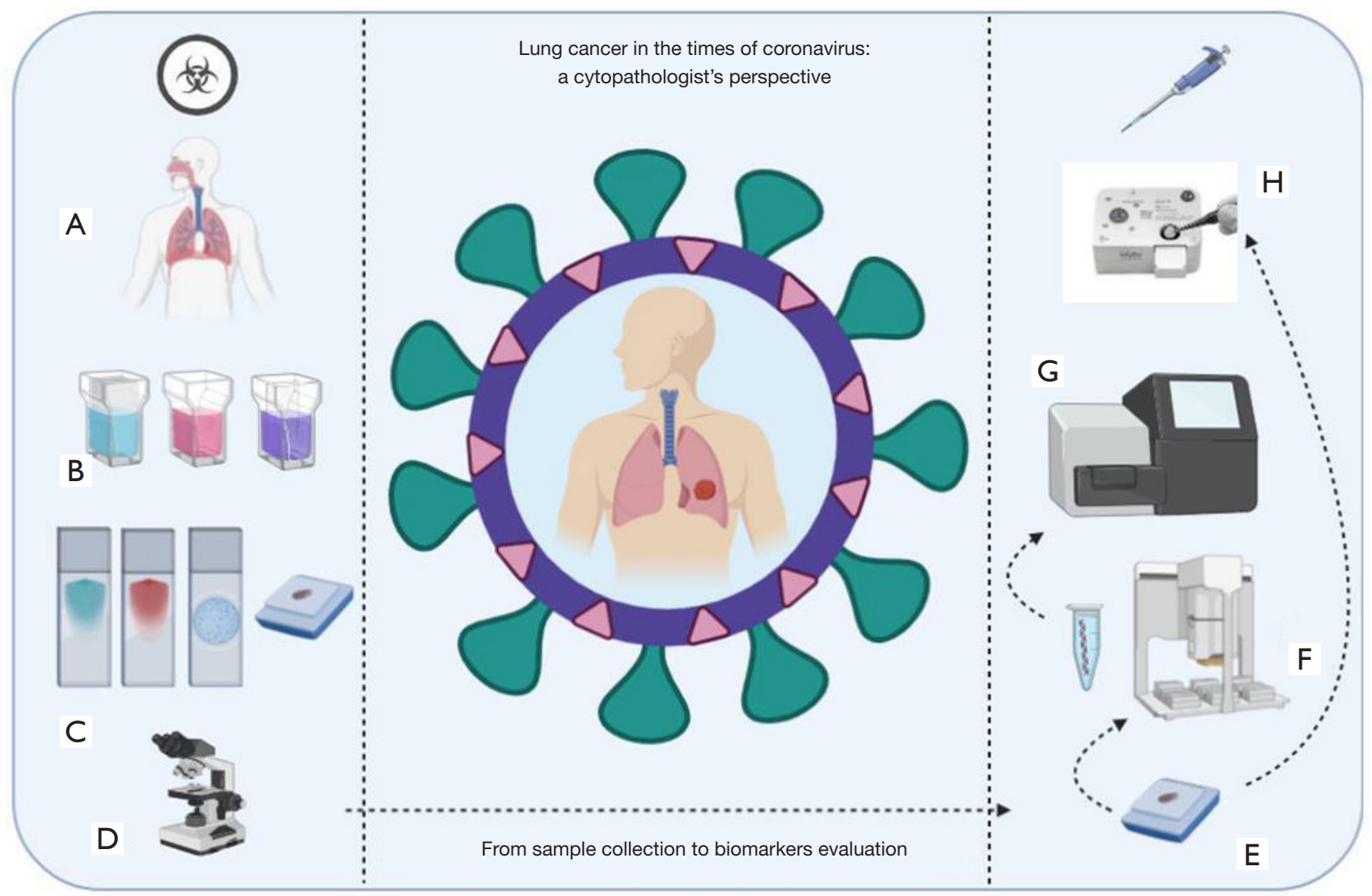

Figure 1 In the times of COVID-19 pandemic, processing pulmonary cytological specimens requires different levels of laboratory biosafety measures (A). As a matter of the facts, rapid on site evaluation, usually performed by using Diff Quik staining (B), and the different cytopreparations (C) protocols should be modified to prevent the transmission of viable SARS-CoV-2, before microscopic evaluation (D). Also molecular pathology required reshaping and reorganizing of the genotyping workflow, to guarantee laboratory staff security. In particular, to reduce hands-on work and to limit the amount of time spent by the laboratory staff to process lung cancer samples (E), timely procedures (F,G) have been replaced by fully automated technologies (H). (Credit: Created with Biorender). COVID-19, coronavirus disease 19; SARS$\mathrm{CoV}-2$, syndrome coronavirus 2 .

pulmonary lesions, as the cells, instead of being smeared, are flushed into a low alcoholic concentration collection medium such as PreservCyt and CytoLyt (Hologic, Inc, Marlborough, MA) and SurePath (BD, Franklin Lakes, NJ) and processed on automated devices (24). Unfortunately, it is uncertain whether these weak alcohol solutions, are adequately inactivating the virus thus that additional precautions, like the use of gloves, may be indicated when preparing and observing LBC slides (24). In order to overcome this issue, a new virus-inactivating method was suggested by the Hologic for all LBC specimens (24). This novel method is based on a preliminary fixation step in $>70 \%$ ethanol, then followed by centrifugation and sequential cell pellet suspension first in CytoLyt and then in PreservCyt (24). This modified technique might increase the amount of fibrin in the background, especially for fineneedle aspiration biopsies, probably related to the sudden fixation of the haemorrhagic material in a large volume of ethanol (24). Due to the increased ethanol concentration, the cells are also smaller and more scattered than in samples processed with the original technique. Although, the distinction between normal, reactive, and atypical cells may be on occasions more difficult, the nuclear details of the neoplastic cells are generally well preserved and the immune 
reactivity of the majority of the cells is also maintained (24).

\section{Biosafety and ROSE}

Respiratory cytological samples can be obtained by a number of sampling procedures that are usually less invasive than the histological approaches (27). Cytological procedures can exploit the possibility to monitor in real time the quality in terms of informativeness of the obtained material (27). In fact, these minimal invasive procedures are much more cost-effective when associated to ROSE (27). This is key since these procedures are not always well tolerated by the patients and reducing the number of passes is mandatory when performing FNA during computed tomography (CT), magnetic resonance imaging (MRI), endoscopy ultrasound (EUS) and endobronchial US (EBUS) (28). ROSE allows not only to check sample adequacy to establish a microscopic diagnosis but also to ensure that tissue material is sufficient to perform ancillary techniques, including immunohistochemistry, microbiology studies, flow cytometry analysis, and molecular assays (29). However, aerosols or droplets may be generated in different steps during the ROSE workflow; in fact, contamination may occur when the tissue material is forced to be expelled from the needle or syringe, when smearing the material, when air-drying the slides, in particular if the smears are agitated by hand. Ideally, these procedures should be performed under class II BSC $(9,17)$. However, such equipment that are available in most pathology laboratories are not commonly found in the radiological or endoscopic suites. Thus that, it is mandatory that the healthcare providers protect themselves with PPE. These latter include eye protection, water-resistant, long-sleeve gown covers, shoe covers, and gloves. EU FFP2 masks or a higher level of protection are used for EBUS transbronchial needle aspiration procedures (17).

Usually, ROSE is performed on air-dried Diff Quik stained smears; during the current healthcare emergency this method may represent a biosafety risk not only for the droplets and aerosol generation from unfixed smears but also for the inherent features of the Diff Quik method (Figure $1 B, C, D)(30)$. This rapid method is a Romanowski group of stains derivate that requires methanol fixation (reagent A), eosin (reagent B) and methylene blue (reagent C) staining. Usually, slides are briefly fixed in $80 \%$ methanol solution reagent A for 20 to 40 seconds, which is not effective in inactivating the SARS-CoV-2 $(16,30)$.
Even longer fixation times (60 to 90 seconds) are not sufficient whereas longer treatments may result into cell damage and fixation artifacts $(16,30)$. As an alternative in most institutions, rapid Pap stain is used; this method requires alcohol fixation of the smears, with sub-sequential hematoxylin, OG-6 and EA-50 treatments, with the excess of staining quickly removed by $1 \%$ acetic acid (30). A better morphological preservation may be obtained by the ultrafast Pap staining, that, however, requires longer times of smear air-drying which makes more common do loss of cellular details. Alternatively, rapid hematoxylin and eosin $(\mathrm{H} \& \mathrm{E})$ staining offers the advantage of immediate alcohol fixation and of high standard of morphological preservation. Another possible solution to Diff Quik stain is the cheap, available, and rapid toluidine blue method, although most pathologists are not familiar with this staining. As a general rule, air-dried specimens should be limited and methanol fixation avoided (30).

\section{BAL and SARS-CoV-2}

Nasopharyngeal and oropharyngeal (NP/OP) swabs are commonly used to detect the SARS-CoV-2 RNA; these upper respiratory tract specimens, however, may yield false negative results, reflecting suboptimal NP/OP tissue sampling (31). Moreover, nasal and pharyngeal cells do not express receptors able to bind SARS-CoV-2; conversely these receptors are abundant on the cell membranes of type I and type II alveolar epithelial cells, explaining a persistent virus detection in lower respiratory tract specimens (32). Thus, according to World Health Organization (WHO) guidelines (33), negative specimens from the upper respiratory tract do not exclude the diagnosis, and additional lower respiratory tract samples are recommended in cases with clinical and radiological suspicion of coronavirus disease 19 (COVID-19) pneumonia (34). Beyond the clinical management of the patients, BAL yields microenvironment relevant information on bronchioles and lung alveoli (35). In particular, a recent study performed single-cell RNA sequencing on BAL cells showing that proinflammatory monocyte-derived macrophages are abundant in the BAL fluid from patients with severe COVID-19 pneumonia, whereas presence of highly clonally expanded CD8+ T cells is associated to a less aggressive disease course (35). From a microscopy point of view, together with generic morphologic features of viral infection such as cytomegaly, syncytia formation, intracytoplasmic and intranuclear 
inclusions, in the COVID-19 BAL specimen, case reports described exuberant plasmacytosis with a large number of activated CD138 positive cells (36) or hemosiderin-laden macrophages identified by specific staining, such as Perls stain or immunocytochemical technique to detect ferric iron (37).

\section{SARS-CoV-2 and molecular predictive pathology}

During the COVID-19 healthcare emergency, elective surgeries and medical procedures scheduled as non-urgent were postponed (18,38-42). In particular, any screening procedure was temporarily suspended, including breast cancer screening program, colonoscopy to investigate positive immunohistochemical fecal occult blood tests, cervical uterine cytology and prostate specific antigen (PSA) screening (11). Similarly, molecular screening of genetically based neoplastic diseases was also postponed (11). In this setting, $B R C A$ gene testing is an exemplificative case. In fact, it was postponed when performed to evaluate the hereditary cancer risk patients' relatives, conversely it was carried out without delays for those patients with ovary neoplasia to evaluate olaparib as a target therapy option for patients harboring alterations for homologous recombination deficiency (HRD) (43). As far as lung cancer predictive biomarker testing is concerned, in our and in other laboratories no significant variation in the total number of tested cases was observed (10). However, molecular laboratory activities required reshaping and reorganizing the genotyping workflow, to guarantee laboratory staff security $(10,44)$. In this scenario, fully automated technologies, requiring minimal hands-on work, were adopted to limit the amount of time spent by the laboratory staff to process lung cancer samples (10). In fact, in our laboratory the standard technology represented by next generation sequencing that requires a long workflow and more than one operator was in almost all cancer cases (92.7\%; 38/41), including lung cancer samples, replaced by the robotic Idylla platform (Biocartis, Mechelen, Belgium) (Figure $1 E, F, G, H)(10)$. A reduction in the number of samples processed was reported only for liquid biopsy testing, reflecting the potential source of transmission, pointing out the need of timely recommendation that should be provided soon to regulate this activity (10). Recently, a multi-centric study investigated the effect of COVID-19 pandemic on lung cancer molecular pathology. Results obtained from 15 European laboratories showed that the number of cases processed during the lockdown $(\mathrm{n}=1,118)$ was very similar to that of a corresponding period in $2019(\mathrm{n}=963)(45)$.

\section{Conclusions}

A number of biosafety measures, mainly procedures equivalent to BSL 2 and 3, are necessary to mitigate the not negligible risk of handling cytopathological samples potentially carrying viable and thus transmissible SARSCoV-2 $(14,15,17)$. Ideally, the needs to inactivate virus and to preserve cyto-preparation quality and morphological details should be balanced (24). As a general rule, on a preliminary fixation step in concentrated ethanol is key to handle body fluids, diagnostic smears and LBC slides and any effort should be taken to limit aerosols or droplets generation in different steps during FNA and ROSE procedures $(24,30)$. Besides the clinical relevance, cytological samples are representative of bronchioles and lung alveoli microenvironment and represent a relevant research tool to better understand COVID-19 pathogenesis (35). This health care emergency also underlines the need to exploit technological advances upgrading molecular pathology laboratory technologies by promoting automation and automatizing the specimen-toreport workflow (10).

\section{Acknowledgments}

Funding: None.

\section{Footnote}

Provenance and Peer Review: This article was commissioned by the editorial office, Translational Lung Cancer Research. The article did not undergo external peer review.

Reporting Checklist: The authors have completed the Narrative Review reporting checklist. Available at http:// dx.doi.org/10.21037/tlcr-20-795

Conflicts of Interest: The authors have completed the ICMJE uniform disclosure form (available at http://dx.doi. org/10.21037/tlcr-20-795). RR serves as an unpaid Editorin-Chief of Translational Lung Cancer Research from June 2019 to May 2022. The authors have no other conflicts of interest to declare. 
Ethical Statement: The authors are accountable for all aspects of the work in ensuring that questions related to the accuracy or integrity of any part of the work are appropriately investigated and resolved.

Open Access Statement: This is an Open Access article distributed in accordance with the Creative Commons Attribution-NonCommercial-NoDerivs 4.0 International License (CC BY-NC-ND 4.0), which permits the noncommercial replication and distribution of the article with the strict proviso that no changes or edits are made and the original work is properly cited (including links to both the formal publication through the relevant DOI and the license). See: https://creativecommons.org/licenses/by-nc-nd/4.0/.

\section{References}

1. Rocco G, Morabito A, Leone A, et al. Management of non-small cell lung cancer in the era of personalized medicine. Int J Biochem Cell Biol 2016;78:173-9.

2. Salto-Tellez M. More Than a Decade of Molecular Diagnostic Cytopathology Leading Diagnostic and Therapeutic Decision-Making. Arch Pathol Lab Med 2018. [Epub ahead of print].

3. Troncone G, Roy-Chowdhuri S. Key Issues in Molecular Cytopathology. Arch Pathol Lab Med 2018;142:289-90.

4. Davidson MR, Gazdar AF, Clarke BE. The pivotal role of pathology in the management of lung cancer. J Thorac Dis 2013;5 Suppl 5:S463-78.

5. Aisner DL, Rumery MD, Merrick DT, et al. Do More With Less: Tips and Techniques for Maximizing Small Biopsy and Cytology Specimens for Molecular and Ancillary Testing: The University of Colorado Experience. Arch Pathol Lab Med 2016;140:1206-20.

6. Clark DP. Seize the opportunity: underutilization of fineneedle aspiration biopsy to inform targeted cancer therapy decisions. Cancer 2009;117:289-97.

7. Jain D, Roy-Chowdhuri S. Molecular Pathology of Lung Cancer Cytology Specimens: A Concise Review. Arch Pathol Lab Med 2018;142:1127-33.

8. Barbareschi M, Barberis M, Buttitta F, et al. Predictive markers in lung cancer: a few hints for the practicing pathologist. Pathologica 2018;110:29-38.

9. Pambuccian SE. The COVID-19 pandemic: implications for the cytology laboratory. J Am Soc Cytopathol
2020;9:202-11.

10. Malapelle U, De Luca C, Iaccarino A, et al. Predictive molecular pathology in the time of COVID-19. J Clin Pathol 2020. [Epub ahead of print].

11. Troncone G, Hofman P. Pathologists and the coronavirus distraction effect. J Clin Pathol 2020. [Epub ahead of print].

12. Health and safety executive advisory committee on dangerous pathogens. The Approved list of biological agents. Secondary the Approved list of biological agents. Available online: www.hse.gov.uk/pubns/misc208.pdf

13. Iwen PC, Stiles KL, Pentella MA. Safety Considerations in the Laboratory Testing of Specimens Suspected or Known to Contain the Severe Acute Respiratory Syndrome Coronavirus 2 (SARS-CoV-2). Am J Clin Pathol 2020;153:567-70.

14. Barbareschi M, Ascoli V, Bonoldi E, et al. Biosafety in surgical pathology in the era of SARS-Cov2 pandemia. A statement of the Italian Society of Surgical Pathology and Cytology. Pathologica 2020;112:59-63.

15. World Health Organization (WHO). Laboratory biosafety guidance related to the novel coronavirus (2019-nCoV). Interim guidance Available online: https://www.who.int/ docs/default-source/coronaviruse/laboratory-biosafetynovel-coronavirus-version-1-1.pdf?sfvrsn=912a9847_2

16. Chen CC, Chi CY. Biosafety in the preparation and processing of cytology specimens with potential coronavirus (COVID-19) infection: Perspectives from Taiwan. Cancer Cytopathol 2020;128:309-16.

17. Centers for disease control and prevention, laboratory biosafety guidelines for handling and processing specimens associated with Coronavirus Disease 2019 (COVID-19). Available online: https://www.cdc.gov/coronavirus/2019ncov/lab/lab-biosafety-guidelines.html

18. Vigliar E, Iaccarino A, Bruzzese D, et al. Cytology in the time of coronavirus disease (covid-19): an Italian perspective. J Clin Pathol 2020. [Epub ahead of print].

19. World Health Organization (WHO). Rational Use of Personal Protective Equipment for Coronavirus Disease 2019 (COVID-19). Interim Guidance, 19 March 2020. WHO, 2020. Available online: https://apps.who.int/iris/ bitst ream/handl e/10665 /33149 8/WHO-2019-nCoVIPCPPE_use-2020.2-eng.pdf17

20. da Cunha Santos G, Saieg MA. Preanalytic specimen triage: Smears, cell blocks, cytospin preparations, 
transport media, and cytobanking. Cancer Cytopathol 2017;125:455-64.

21. Miller JM, Astles R, Baszler T, et al. Guidelines for safe work practices in human and animal medical diagnostic laboratories. Recommendations of a CDCconvened, Biosafety Blue Ribbon Panel. MMWR Suppl 2012;61:1-102.

22. Gosney JR, Hofman P, Troncone G, et al. Cellular pathology in the COVID-19 era: a European perspective on maintaining quality and safety. J Clin Pathol 2020. [Epub ahead of print].

23. Sun J, Xiao J, Sun R, et al. Prolonged Persistence of SARS-CoV-2 RNA in Body Fluids. Emerg Infect Dis 2020;26:1834-8.

24. Rossi ED, Fadda G, Mule A, et al. Cytologic and histologic samples from patients infected by the novel coronavirus 2019 SARS-CoV-2: An Italian institutional experience focusing on biosafety procedures. Cancer Cytopathol 2020;128:317-20.

25. Gabriel C, Achten R, Drijkoningen M. Use of liquid-based cytology in serous fluids: a comparison with conventional cytopreparatory techniques. Acta Cytol 2004;48:825-35.

26. Bellevicine C, Malapelle U, Vigliar E, et al. Epidermal growth factor receptor test performed on liquid-based cytology lung samples: experience of an academic referral center. Acta Cytol 2014;58:589-94.

27. Jain D, Allen TC, Aisner DL, et al. Rapid On-Site Evaluation of Endobronchial Ultrasound-Guided Transbronchial Needle Aspirations for the Diagnosis of Lung Cancer: A Perspective From Members of the Pulmonary Pathology Society. Arch Pathol Lab Med 2018;142:253-62.

28. Aziz F. Endobronchial ultrasound-guided transbronchial needle aspiration for staging of lung cancer: a concise review. Transl Lung Cancer Res 2012;1:208-13.

29. Bellevicine C, Malapelle U, Vigliar E, et al. How to prepare cytological samples for molecular testing. J Clin Pathol 2017;70:819-26.

30. Asiry S, Fatyan A, Matloob A, et al. Safety during crisis: Rapid on-site evaluation at the time of COVID-19 pandemic. Diagn Cytopathol 2020;48:813-5.

31. Winichakoon P, Chaiwarith R, Liwsrisakun C, et al. Negative Nasopharyngeal and Oropharyngeal Swabs Do Not Rule Out COVID-19. J Clin Microbiol 2020;58:e00297-20.

32. Valan AB, Sture C. Negative nasopharyngeal swabs early in the course of COVID-19. Tidsskr Nor Laegeforen 2020. doi: 10.4045/tidsskr.20.0356.

33. World Health Organization (WHO) Coronavirus disease (COVID-19) technical guidance: early investigation protocol Available from WHO, Geneva (2020). Available online: https://www.who.int/emergencies/diseases/novelcoronavirus-2019/technical-guidance/early-investigation

34. Gualano G, Musso M, Mosti S, et al. Usefulness of bronchoalveolar lavage in the management of patients presenting with lung infiltrates and suspect COVID19-associated pneumonia: A case report. Int J Infect Dis 2020;97:174-6.

35. Liao M, Liu Y, Yuan J, et al. Single-cell landscape of bronchoalveolar immune cells in patients with COVID-19. Nat Med 2020;26:842-44.

36. Giani M, Seminati D, Lucchini A, et al. Exuberant Plasmocytosis in Bronchoalveolar Lavage Specimen of the First Patient Requiring Extracorporeal Membrane Oxygenation for SARS-CoV-2 in Europe. J Thorac Oncol 2020;15:e65-6.

37. Drak Alsibai K. Detection of Hemosiderin-Laden Macrophages in Bronchoalveolar Lavage Fluid of COVID-19 Patients: Is Perls Stain a Potential Indicator of Oxidative Alveolar Damage? Acta Cytol 2020. [Epub ahead of print].

38. Kaye K, Paprottka F, Escudero R, et al. Elective, Nonurgent Procedures and Aesthetic Surgery in the Wake of SARS-COVID-19: Considerations Regarding Safety, Feasibility and Impact on Clinical Management. Aesthetic Plast Surg 2020;44:1014-42.

39. Stahel PF. How to risk-stratify elective surgery during the COVID-19 pandemic? Version 2. Patient Saf Surg 2020;14:8.

40. Troncone G. Thyroid cytology in the times of coronavirus. Diagn Cytopathol 2020. [Epub ahead of print].

41. Bellevicine C, Vigliar E, Troncone G. Thyroid FNA in the time of coronavirus: The interventional cytopathologist point of view. Cancer Cytopathol 2020;128:589.

42. Rossi ED, Pantanowitz L. International perspectives: Impact of the COVID-19 pandemic on cytology. Cancer Cytopathol 2020;128:307-8.

43. Minucci A, Scambia G, Santonocito C, Concolino P, Urbani A. BRCA testing in a genomic diagnostics referral center during the COVID-19 pandemic. Mol Biol Rep 2020;47:4857-60.

44. Bardelli A. Coronavirus lockdown: What I learnt when I 
shut my cancer lab in 48 hours. Nature 2020. [Epub ahead of print].

45. Malapelle U, Pisapia P, Iaccarino A, et al. Predictive molecular pathology in the time of coronavirus disease (COVID-19) in Europe. J Clin Pathol 2020. [Epub ahead of print].

Cite this article as: Pisapia P, Malapelle U, Salatiello M, Rosell

$\mathrm{R}$, Troncone G. A narrative review of lung cancer cytology in the times of coronavirus: what physicians should know. Transl Lung Cancer Res 2020;9(5):2074-2081. doi: 10.21037/tlcr-20-795 